\title{
Internal and external catalysis in boronic ester networks
}

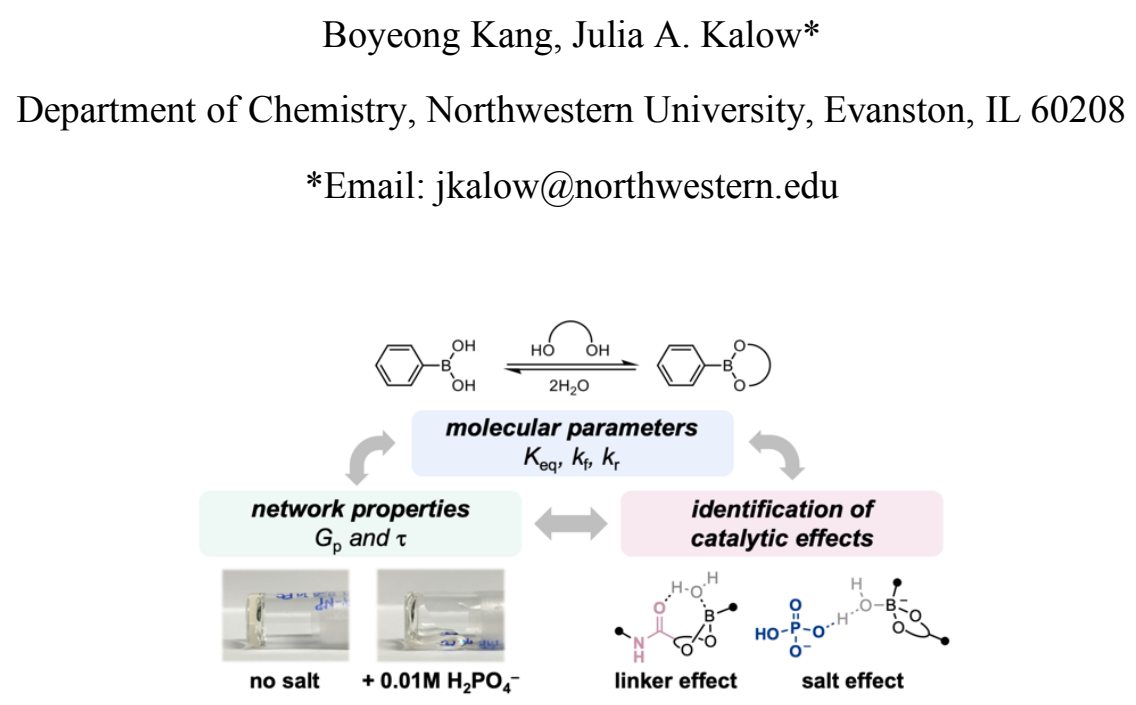

\section{Summary}

By comparing the molecular kinetics of the dynamic boronic ester bond to the mechanical properties of boronic ester gels, we find that the amide groups, commonly used to link diols to polymers, and anions in buffers act as internal and external catalysts.

\begin{abstract}
In dynamic materials, the reversible condensation between boronic acids and diols provides adaptability, self-healing ability, and responsiveness to small molecules and $\mathrm{pH}$. Recent work has shown that the thermodynamics and kinetics of bond exchange determine the mechanical properties of dynamic polymer networks. However, prior studies have focused on how structural and environmental factors influence boronic acid-diol binding affinity, and design rules for tuning the kinetics of this dynamic bond are lacking. In this work, we investigate the effects of diol (or polyol) structure and salt additives on the rate of bond exchange, binding affinity, and the mechanical properties of the corresponding polymer networks. To better mimic the environment of polymer networks in our small-molecule model systems, we incorporated proximal amide groups, which are used to conjugate diols to polymers, and included salts commonly found in buffers. Using one-dimensional selective exchange spectroscopy (1D EXSY), we find that both proximal amides and buffering anions induce significant rate acceleration consistent with internal and external catalysis, respectively. This rate acceleration is reflected in the stress relaxation of gels formed using PEG modified with different alcohols, and in the presence of salts containing acetate or phosphate. These findings contribute to the fundamental understanding of the boronic ester dynamic bond and offer new molecular strategies to tune the macromolecular properties of dynamic materials.
\end{abstract}




\section{Introduction}

The reversible condensation between boronic acids and diols has been applied to sensors, ${ }^{1-5}$ drug delivery, ${ }^{5,6}$ and dynamic hydrogels ${ }^{3,5,7-21}$ due to its biocompatibility, selectivity, and reactivity under ambienttemperature aqueous conditions. Among dynamic covalent chemistries, boronic acid-diol exchange offers the fastest uncatalyzed rates, enabling rapid responses to physical and chemical stimuli. ${ }^{1,22,23}$ In polymer networks, incorporating boronic acids and diols within polymer chains or at end groups endows self-healing behavior, stimuli responsiveness, and viscoelasticity. Experiment, simulations, and theory have revealed that the macroscopic properties of dynamic networks are highly dependent on the thermodynamics and kinetics of the reversible crosslink. ${ }^{24-40}$ While thermodynamics (binding affinity) determines the stiffness of the polymer network, the important dynamic behaviors such as self-healing and viscoelasticity are governed by the kinetics of the reversible reaction (Figure 1a). Therefore, to better control and design boronic ester-based materials for specific applications, it is essential to understand the molecular factors that influence both kinetics and thermodynamics of this dynamic bond.

The esterification and hydrolysis of boronic acids and esters occur via multi-step mechanisms and are sensitive to many factors: $\mathrm{pKa}$ of the boronic acid and diol, sterics and dihedral angle of the diol, $\mathrm{pH}$ of the solution, and buffer composition and concentration (Figure 1b). ${ }^{41-43}$ As a result, the reaction landscape varies significantly depending on the choice of the boronic acid and diol partners as well as the reaction conditions. Efforts to understand how those factors affect the thermodynamics of the dynamic bond have focused on maximizing the binding affinity $\left(K_{\text {eq }}\right)$ at physiological $\mathrm{pH} .{ }^{9,42,44-49} \mathrm{In}$ particular, those studies have introduced new classes of boronic acids with high binding affinities, such as Wulff-type boronic acid and benzoxaborole. ${ }^{9,44,50}$ In contrast, studies investigating the kinetics of this dynamic reaction are largely motivated by boronic acid-based receptors and sensors, and thus rely on specific model diols to elucidate the speciation and pathways of the complex mechanism (see Electronic Supplementary Information (ESI), Table S1). ${ }^{51-64}$ Unlike the extensive structure-reactivity relationships available for boronic acid-diol binding affinity, design principles to tune the kinetics of the reaction have not been systematically developed. Furthermore, factors of particular relevance to polymer networks, such as the conjugation chemistry used to attach the dynamic bond to polymers or commonly used buffers for hydrogels, are not typically included in small-molecule model studies. In the only study to directly compare free and polymerbound sugars, Auzély-Velty noted differences in their binding constants with boronic acids. ${ }^{28}$ 
(a)

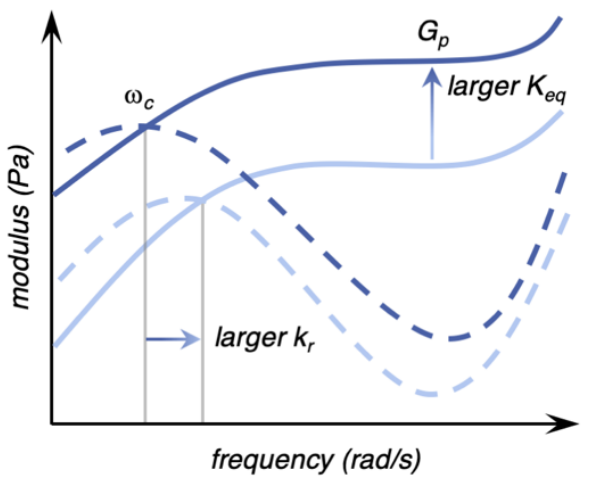

(b)
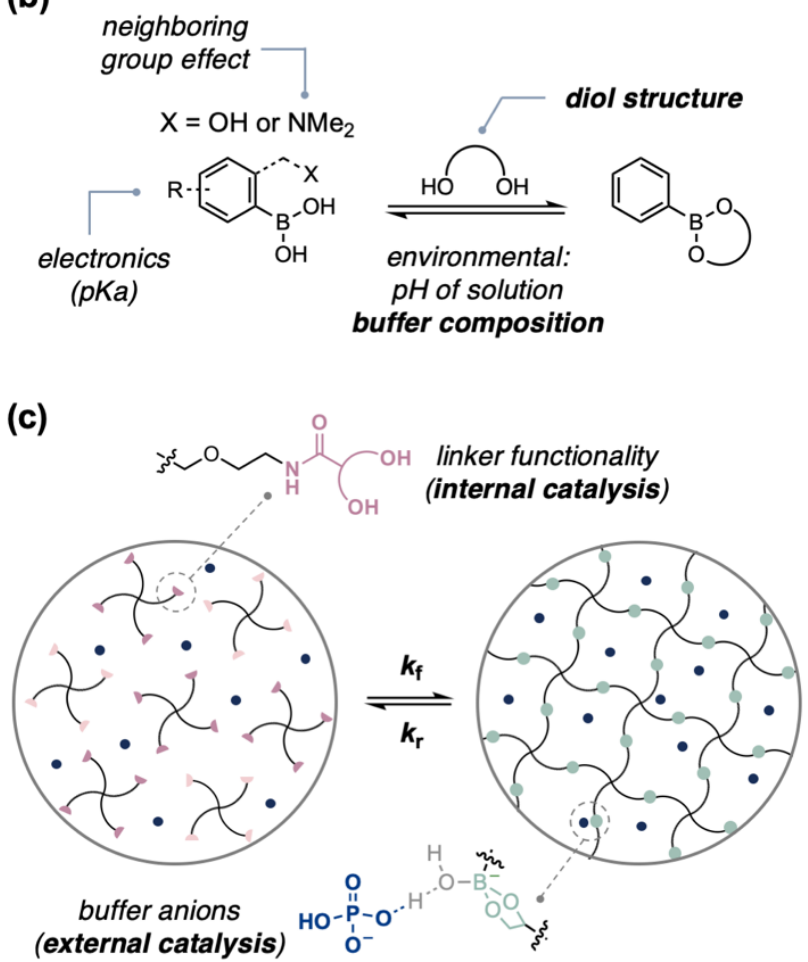

Figure 1. (a) Translation of molecular parameters into macroscopic network properties. The binding constant $\left(K_{\text {eq }}\right)$ relates to the rubbery plateau modulus $\left(\mathrm{G}_{\mathrm{p}}\right)$ while the rate of dissociation $\left(k_{\mathrm{r}}\right)$ determines the viscoelasticity of the network. (b) Summary of factors affecting the boronic acid-diol reversible condensation. This work focuses on the effect of diol structure and salts on the thermodynamics and kinetics of the boronic acid-ester equilibrium. (c) This work uncovers internal catalysis in boronic ester networks by the linker used to conjugate diol to polymer end groups, and external catalysis by buffering anions.

Here, we report the effect of diol structure and salt additives on both kinetics and thermodynamics of the reversible boronic acid-diol condensation and their impact on the mechanical properties of boronic ester networks. We measured the exchange kinetics and binding affinity between different diols (or polyols) and phenylboronic acid (PBA) by one-dimensional selective exchange spectroscopy (1D EXSY) and ${ }^{1} \mathrm{H}$ NMR. We then synthesized star polymers with analogous diol/polyol and boronic acid end groups and measured 
the mechanical properties of the resulting gels using shear rheology. Through these measurements, we uncovered two new factors that affect the mechanics of boronic ester networks: the presence of amide groups proximal to the diol, and the composition of the buffer (Figure 1c). We showed that amide groups used to conjugate diols to polymers act as internal catalysts via a solvent-insertion mechanism, ${ }^{65}$ accelerating both esterification and hydrolysis. Additionally, we observed salt-dependent rate constants correlated to the $\mathrm{pKa}$ of the anion's conjugate acid (Figure 1c). These changes in molecular thermodynamics and kinetics are translated into gels with a wide range of mechanical properties, with shear moduli ranging from $1.8 \mathrm{kPa}$ to $27 \mathrm{kPa}$ and relaxation times from $0.1 \mathrm{~s}$ to $571 \mathrm{~s}$, based only on changes in diol structure or salt additive. Our results inform new design parameters that can be used to tune boronic ester-based dynamic materials and complement existing studies that have focused on the boronic acid partner. Furthermore, these findings emphasize the importance of designing small-molecule model systems that capture essential components of the polymer network when relating molecular parameters to network properties.

\section{Results and discussion}

\section{Effect of diol structure on kinetics of the dynamic covalent reaction}

To study the effect of diol structure on boronic ester formation and hydrolysis, we selected a representative 1,2-diol, 1,3-diol, triol, and polyol with varying degrees of flexibility and steric encumbrance (Figure 2a, "simplified alcohols"). The reaction kinetics of the reversible condensation between PBA and each alcohol were studied by using 1D EXSY. EXSY NMR detects magnetization transfer via chemical exchange, allowing quantitative measurement of the rate of dynamic processes occurring slower than the NMR time scale. In 1D EXSY, a peak of interest is selectively excited, and the intensities of the irradiated and exchanged peaks are monitored as a function of mixing time. Thus, a series of measurements with different mixing times tracks the magnetization transfer between exchanging species over time. When the mixing times are long enough for the exchange to reach equilibrium, the relative intensities of the irradiated and exchanging peaks become constant.

The dynamic reaction was studied under pseudo-first order conditions by dissolving PBA in a mixture of acetone- $d_{6}$ and $\mathrm{D}_{2} \mathrm{O}$ (1.2:1) with excess alcohol (10 equiv.). Under these conditions, we confirmed that the ortho-protons of the boronic acid and ester are well resolved by ${ }^{1} \mathrm{H}$ NMR and thus can be selectively irradiated (Figure 2b; see ESI for additional examples). In the 1D EXSY experiment, the ortho-protons of PBA were irradiated, and the integrated peaks of the acid and the ester were converted to concentrations for each mixing time using an internal standard, following a literature procedure (Figure 2c). ${ }^{66}$ The decrease of in acid concentration with respect to mixing time was fit to a first-order reversible rate law to obtain pseudo-first-order rate constants for the forward (esterification, $k_{\mathrm{obs}, \mathrm{f}}$ ) and reverse (hydrolysis, $k_{\mathrm{obs}, \mathrm{r}}$ ) reactions. These measurements were repeated at several temperatures to determine the activation energies for esterification and hydrolysis (Figure 2d, see ESI for details). 
(a)
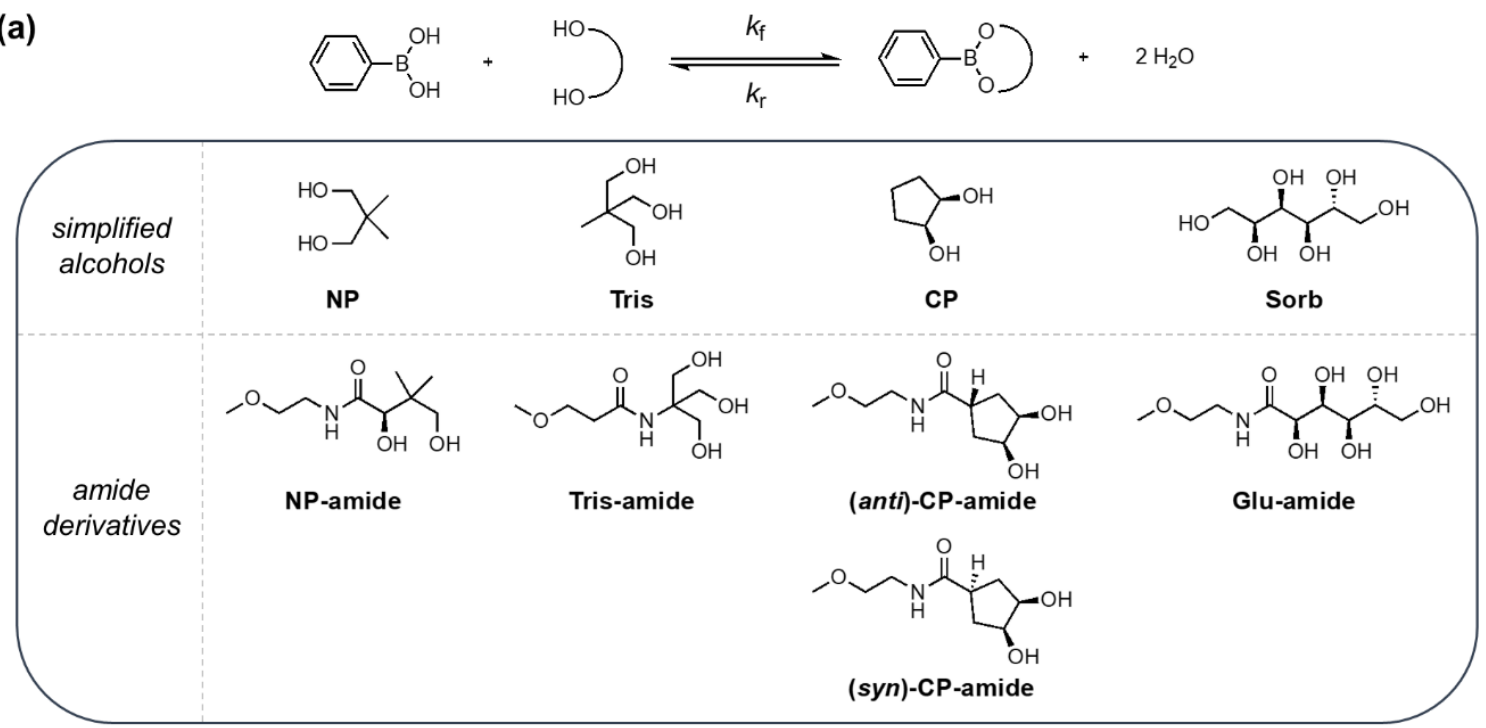

(b)

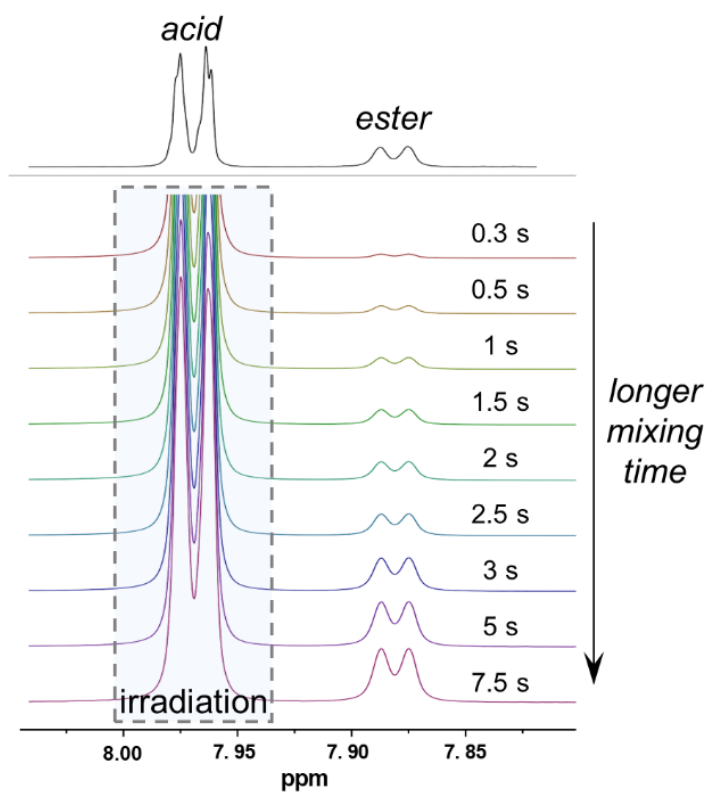

(c)

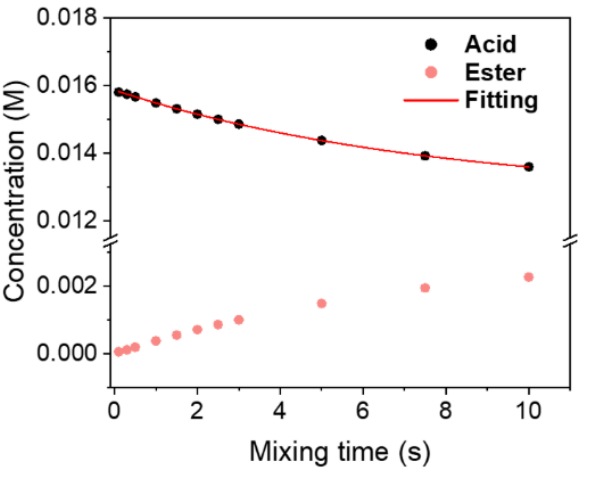

(d)

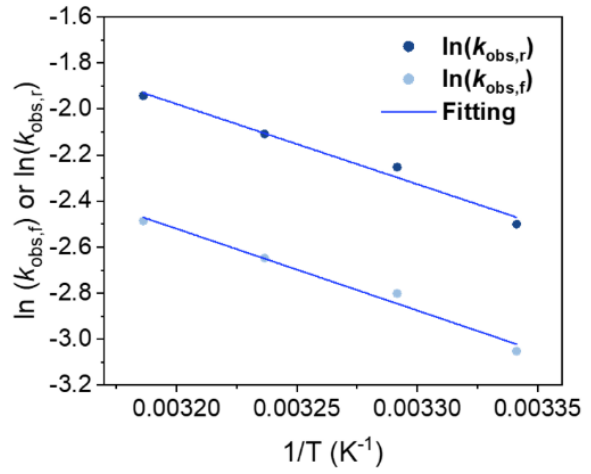

Figure 2. (a) The reversible condensation of PBA and alcohols, and the structures of the alcohols used in this study. (b) Representative ${ }^{1} \mathrm{H}$ NMR (top, at equilibrium) and 1D EXSY with different mixing times (bottom) with PBA and NP at $25^{\circ} \mathrm{C}$. The peak at $7.97 \mathrm{ppm}$ (ortho-protons of PBA) is selectively irradiated, and the integrations of the acid and the ester peak at various mixing times were extracted. (c) Analysis of 1D EXSY data. The concentration change of PBA over mixing time was fitted with a first-order reversible rate law. (d) Arrhenius plot using the pseudo-first-order rate constants at different temperatures to calculate the activation energy from the slope. 
The measured kinetic parameters and equilibrium constants for each alcohol are summarized in Table 1; activation energies are reported in Table S3. As has been previously reported, ${ }^{43}$ the flexible 1,3-diols like neopentylglycol (NP) exhibited a significantly lower binding affinities for PBA compared to cis-1,2cyclopentanediol (CP), which has a locked geometry that favors ester formation. Compared to all other alcohols studied, CP exhibits a slower esterification rate, likely due to the steric demands of the secondary alcohols, and a dramatically slower hydrolysis rate that could not be measured directly by EXSY. Increasing the number of $\mathrm{OH}$ groups also enhanced the binding affinity $(\mathbf{N P}<$ Tris $<$ Sorb). As the functionality increases, the rate of esterification increases, and the rate of hydrolysis decreases, albeit to a lesser extent.

Table 1. Equilibrium and rate constants for the reversible esterification/hydrolysis of PBA with various alcohols. $^{a}$

\begin{tabular}{|c|c|c|c|c|c|}
\hline Alcohols & $K_{\text {eq }}$ & $k_{\mathrm{obs}, \mathrm{f}}\left(\mathrm{s}^{-1}\right)$ & $k_{\text {obs,r }}\left(\mathrm{s}^{-1}\right)$ & $\boldsymbol{K}_{\text {rel, }, f^{d}}$ & $k_{\text {rel, }, r^{d}}$ \\
\hline NP & $0.577( \pm 0.009)$ & $0.0473( \pm 0.0004)$ & $0.082( \pm 0.001)$ & & \\
\hline Tris & $1.389( \pm 0.006)$ & $0.1100( \pm 0.0003)$ & $0.0792( \pm 0.0003)$ & & \\
\hline Sorb & $7.4( \pm 0.6)$ & $0.23( \pm 0.01)$ & $0.031( \pm 0.002)$ & & \\
\hline $\mathbf{C P}$ & $15^{b}( \pm 2)$ & $0.0216( \pm 0.0004)$ & $0.0014^{c}( \pm 0.0002)$ & & \\
\hline Tris-amide & $0.182( \pm 0.006)$ & $2.20( \pm 0.04)$ & $12.1( \pm 0.3)$ & 20 & 150 \\
\hline NP-amide & $0.877( \pm 0.005)$ & $0.327( \pm 0.001)$ & $0.373( \pm 0.002)$ & 6.9 & 4.5 \\
\hline (syn)-CP-amide & $1.45( \pm 0.02)$ & $0.210( \pm 0.002)$ & $0.145( \pm 0.002)$ & 9.7 & 100 \\
\hline (anti)-CP-amide & $5.4( \pm 0.4)$ & $0.107( \pm 0.006)$ & $0.020( \pm 0.001)$ & 5.0 & 14 \\
\hline Glu-amide & $6.4( \pm 0.5)$ & $0.70( \pm 0.03)$ & $0.110( \pm 0.008)$ & 3.0 & 3.5 \\
\hline
\end{tabular}

${ }^{a}$ Pseudo-first-order rate constants were determined using $1 \mathrm{D}$ EXSY at $25{ }^{\circ} \mathrm{C}$ with $19 \mathrm{mM}$ PBA in acetone- $\mathrm{d}_{6}: \mathrm{D}_{2} \mathrm{O}$ (1.2:1) and 10 equiv. alcohol. Dimethyl sulfone or ethylene carbonate was used as an internal standard. Equilibrium constants were determined from the forward and reverse rate constants at equilibrium $\left(K_{\text {eq }}=k_{\text {obs, } f} / k_{\mathrm{obs}, \mathrm{r}}\right) .{ }^{b}$ Estimated using $K_{\text {eq }}=\left(\left[\right.\right.$ ester] $\left.\left[\mathrm{H}_{2} \mathrm{O}\right]^{2}\right) /([$ acid] $[$ diol] $])$ because hydrolysis was too slow for the rate constant to be extracted by fitting; see ESI for details. ${ }^{c}$ Estimated from $K_{\text {eq }}$ using $k_{\mathrm{obs}, \mathrm{r}}=k_{\mathrm{obs}, \mathrm{f}} / K_{\text {eq. }}{ }^{\mathrm{d}}$ Calculated by $k_{\mathrm{obs}}$ (amide derivatives) $/ k_{\mathrm{obs}}$ (simplified alcohols).

\section{Network properties do not correlate to molecular parameters of simplified alcohols}

We next synthesized star polymers with end groups that contain the structures of the simplified alcohols (Figure 3a; see ESI for synthetic details). Using amide coupling, 4-arm PEG-carboxylate and amine $\left(\mathrm{M}_{\mathrm{w}}\right.$ $5 \mathrm{kDa}$ ) were conjugated to amines or acids containing Tris, CP, and PBA substructures to generate PEGTris, PEG-CP, and PEG-PBA. PEG-CP end groups represent a mixture of diastereomers (4:1 anti:syn; see ESI for assignment). PEG-NP and PEG-Glu were obtained by ring-opening of pantolactone and gluconolactone by 4-arm PEG amine. Each alcohol-terminated polymer was mixed with PEG-PBA in a $1: 1$ ratio to form a $10 \mathrm{w} / \mathrm{v} \%$ solution in propylene carbonate containing $1 \%(\mathrm{v} / \mathrm{v}) \mathrm{D}_{2} \mathrm{O}$. This solvent mixture was required based on the low binding constants of some of the alcohols tested, which preclude gelation in fully aqueous environments. The gels were characterized by oscillatory shear rheology within the linear viscoelastic regime to determine the rubbery plateau modulus $\left(G_{\mathrm{p}}\right.$, Figure $\mathbf{3 b}$; see ESI for amplitude sweeps). 
(a)

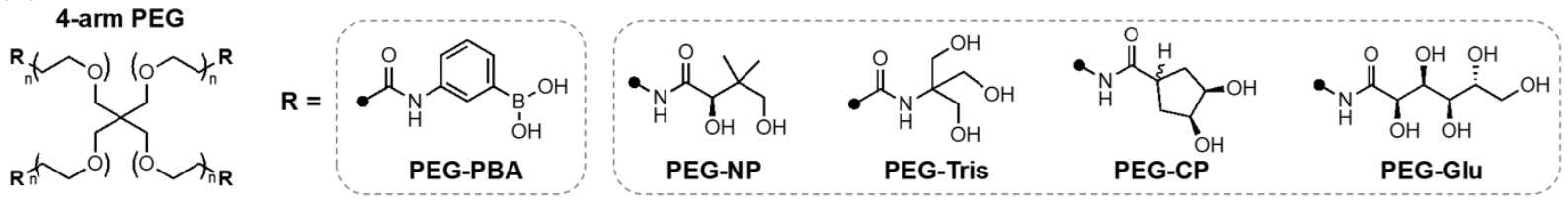

(b)

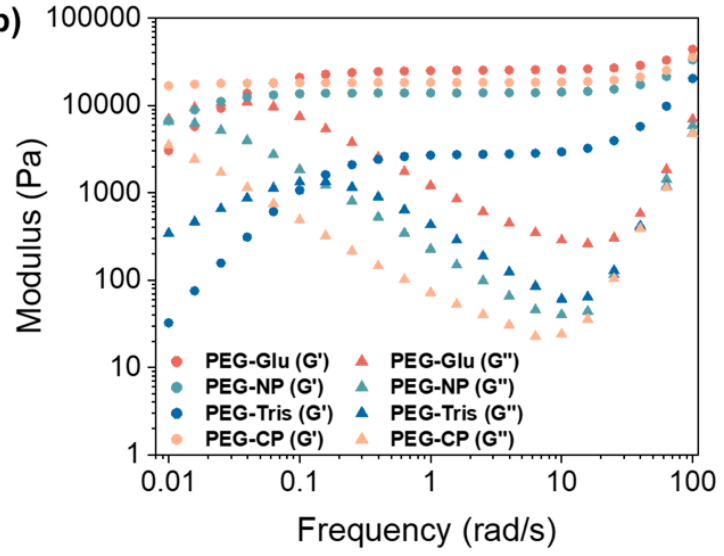

(d)

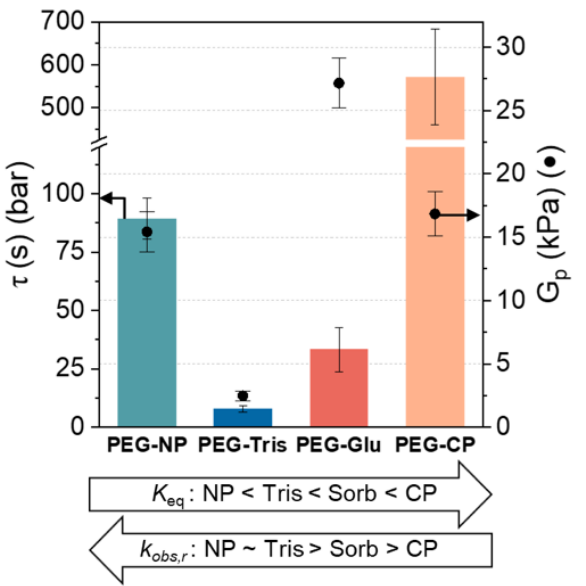

(c)

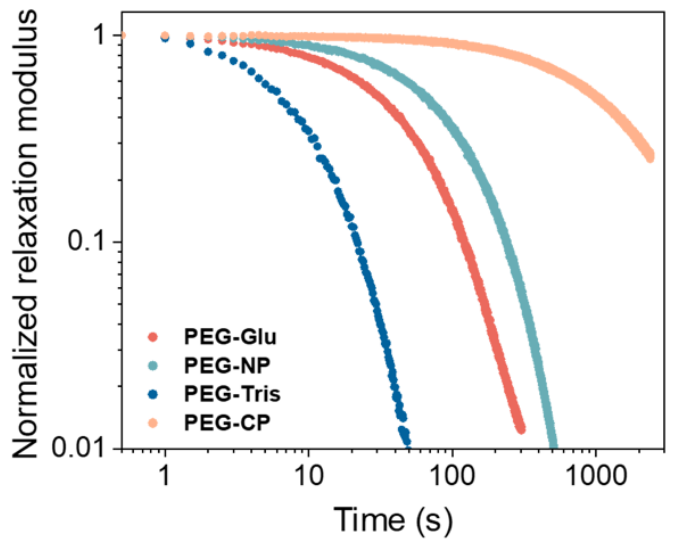

(e)

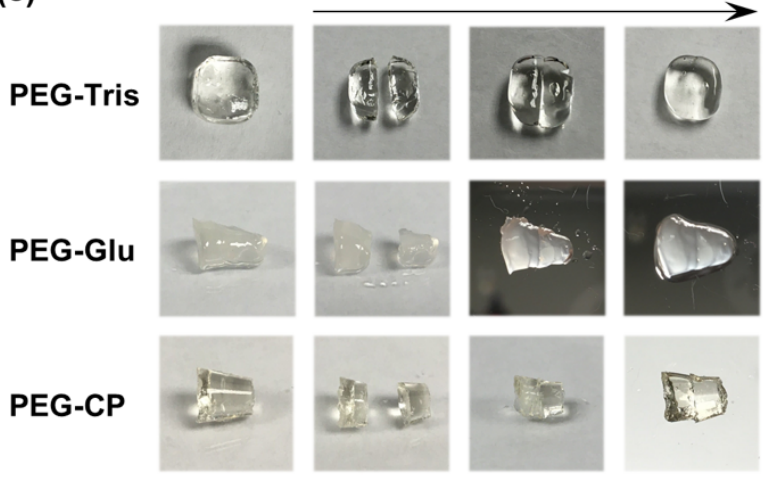

Figure 3. Mechanical and self-healing properties of the gels formed from 4-arm PEG-PBA and PEGalcohol $\left(1: 1,10 \mathrm{w} / \mathrm{v} \%\right.$ in propylene carbonate with $\left.1 \% \mathrm{D}_{2} \mathrm{O}\right)$. (a) Chemical structures of modified PEG polymers. PEG-CP contains two diastereomers in a 4:1 ratio (anti:syn). (b) Frequency sweeps at 5\% strain obtained by oscillatory shear rheology. (c) Normalized stress relaxation profiles following $5 \%$ shear step strain. (d) Calculated $\tau$ for the different gels (bar graph, left axis) and their rubbery plateau moduli, $G_{\mathrm{p}}$ (black dot, right axis). The $\tau$ values, the time required for the relaxation modulus to reach $1 / \mathrm{e}$ of its initial value, were obtained by fitting the stress relaxation data with a Maxwell model. Mechanical data are the average of 3 samples; error is the standard deviation of the mean. (e) Photographs of PEG-Tris, PEG-Glu and PEG-CP gels undergoing self-healing.

In a dynamic network, the number of elastically active polymer strands, which determines $G_{\mathrm{p}}$, is dictated by the equilibrium constant $\left(K_{\mathrm{eq}}\right)$ of the crosslink. Tibbitt recently modified the phantom network model to directly relate $G_{\mathrm{p}}$ to $K_{\mathrm{eq}}$ in dynamic networks and demonstrated the validity of this dynamic model in a boronic ester hydrogel. ${ }^{33}$ Based on the $K_{\text {eq }}$ values obtained for the simplified alcohol in Table 1, we expected the relative rubbery plateau moduli of the corresponding gels to follow the trend PEG-NP $<$ PEG- 
Tris $<$ PEG-Glu $<$ PEG-CP. However, we observed that the moduli followed the trend PEG-Tris $<<$ PEG-NP $<$ PEG-CP $<$ PEG-Glu (Figure 3d).

To relate the kinetics of the dynamic bond and the viscoelasticity of the gels, we applied a step strain (5\%) to the gels and measured the stress relaxation over time (Figure 3c). These data were fit to a Maxwell model to obtain the characteristic relaxation time $\tau$, which were in good agreement with the crossover frequencies $\left(\omega_{c}\right)$ observed in Figure 3b, where experimentally accessible (Table S9). The theoretical foundation established by Semenov and Rubinstein revealed an inverse relationship between $\tau$ and the dissociation rate of the crosslink $\left(\tau \propto 1 / k_{\mathrm{obs}, \mathrm{r}}\right),{ }^{31,32}$ and experimental studies have confirmed this relationship. ${ }^{24-30,33,34}$ If the measured $k_{\mathrm{obs}, \mathrm{r}}$ values of the corresponding simplified alcohols correlate to the bulk relaxation time, we would expect to observe the trend PEG-NP $\sim$ PEG-Tris $<$ PEG-Glu $<<$ PEG-CP. However, the observed trend was PEG-Tris $<$ PEG-Glu $<$ PEG-NP $<<$ PEG-CP (Figure 3d).

Analogously, the rate of self-healing ability in the network should be determined by the rate of crosslink formation $\left(k_{\mathrm{obs}, \mathrm{f}}\right) .{ }^{27,36,37}$ We qualitatively compared self-healing of PEG-Tris, PEG-Glu and PEG-CP gels by cutting them in half and putting them back together (Figure 3e). After $2 \mathrm{~h}$, no fracture was detectable within the PEG-Tris gel, showing complete self-healing, whereas the fracture was smoothed but visible in the PEG-Glu gel and clearly visible in PEG-CP gel (PEG-CP < PEG-Glu < PEG-Tris). Based on the measured $k_{\text {obs,f }}$ trend, we expect self-healing to occur fastest for PEG-Glu and slowest for PEG-CP. Taken together, the discrepancies between the small-molecule parameters and the mechanical properties of the networks led us to re-evaluate the use of simplified alcohols as model compounds.

\section{Internal catalysis by the proximal amide}

The key structural difference between the simplified alcohols in Figure 2a and the crosslinkers in the gel is the presence of amide groups used for conjugation. Previously, our lab has taken advantage of photoswitchable Lewis basic groups proximal to the boronic ester to modulate the kinetics and thermodynamics of its dynamic covalent reactions. ${ }^{8,13,67}$ Anslyn has shown that the basic aminomethyl group in Wulff-type boronic acids catalyzes the addition and elimination of alcohols and water. ${ }^{52,68}$ However, analogous internal catalytic effects have not been shown for the diol component of this dynamic covalent reaction. We hypothesized that the amide groups introduced for polymer functionalization perturb the rate and equilibrium constants of the esterification and hydrolysis reactions, through inductive effects, direct coordination to the boron center, ${ }^{69,70}$ or internal catalysis. ${ }^{40,71}$

To test this hypothesis, we synthesized amide derivatives of each alcohol (Figure 1a, "amide derivatives") and measured the kinetics of their dynamic reactions with PBA using 1D EXSY (Table 1). Indeed, we observed dramatic increases in both esterification and hydrolysis rates for the amide derivatives compared to their simplified alcohol counterparts. These increases ranged from modest (Sorb vs. Glu-amide, $\sim 3$-fold increase in $k_{\mathrm{obs}, \mathrm{f}}$ and $k_{\mathrm{obs}, \mathrm{r}}$ ) to an order of magnitude or greater (Tris vs. Tris-amide, 20-fold increase in $k_{\mathrm{obs}, \mathrm{f}}$ and 150 -fold increase in $k_{\text {obs,r }}$ ). With Glu-amide, we also observed by EXSY the appearance of a new peak that undergoes exchange with both acid and ester (Figure S18). When the boronic acid peak is irradiated at $25{ }^{\circ} \mathrm{C}$, only $5 \%$ conversion to this species is observed, but it becomes a major product when the EXSY experiments are performed at lower temperature. We propose that this species is an intermediate, which is not observed for any of the other alcohols, and may affect the accuracy of the measured rate constants for Glu-amide. DFT calculations by Auzély-Velty suggest that there are at least five different binding modes between PBA and a ring-opened gluconolactone with similar energies; however, these calculations did not include an amide group. ${ }^{28}$ 
The rate acceleration attributed to the amide group could arise from inductive effects, neighboring-group effects, or a combination. To obtain insight into the relative contributions of each effect, we prepared the anti and syn diastereomers of CP-amide and separately measured the reaction kinetics with PBA. Relative to unfunctionalized $\mathbf{C P}$, the minor isomer of $\mathbf{C P}$-amide ( $\mathrm{syn}$ ) experiences more significant rate acceleration (9.7-fold for esterification and 100-fold for hydrolysis) than the major isomer (anti) (5.0-fold and 14-fold, respectively) (Table 1). In (syn)-CP-amide, the amide carbonyl is on the same face of the cyclopentane ring as the diol and is thus well positioned to activate the diol nucleophile when it attacks the boronic acid as an intramolecular base catalyst (Figure 4a). Similarly, in the syn isomer, the amide carbonyl can activate water when it attacks the boronic ester during hydrolysis. In contrast, in (anti)-CP-amide, the carbonyl is on the opposite face of the cyclopentane ring, which prevents any intramolecular catalysis (Figure $4 \mathbf{b}$ ). Nevertheless, the modest rate acceleration in the anti isomer suggests that intermolecular catalysis, inductive effects, and/or conformational effects can accelerate both esterification and hydrolysis relative to unsubstituted CP. Both $\mathrm{C}$ - and $\mathrm{N}$-linked amides are inductively electron-withdrawing, which is expected to accelerate the hydrolysis of the boronic ester. ${ }^{72}$

(a) (syn)-CP-amide
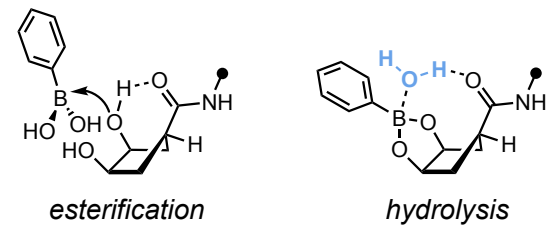

(b) (anti)-CP-amide
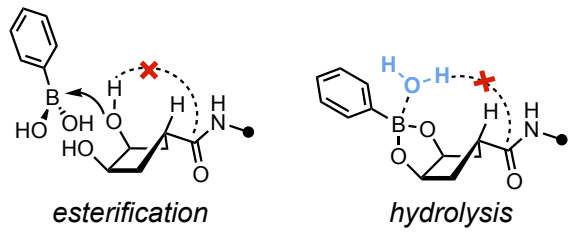

Figure 4. Proposed mode of activation by the amide carbonyl on CP-amide diastereomers during esterification and hydrolysis, for (syn)-CP amide (a) and (anti)-CP-amide (b). In the syn isomer, the amide carbonyl is on the same face of the ring as the diols, thus can activate incoming nucleophiles (i.e. alcohols and water) towards attack at the boron center.

To provide additional evidence for the participation of the amide group, the interaction between boron and the amide group was studied by ${ }^{11} \mathrm{~B}$ NMR. Previously, Anslyn used ${ }^{11} \mathrm{~B}$ NMR to show that Wulff-type boronic esters in alcohol or water form solvent-inserted tetrahedral species that appear at 8-12 ppm (Figure 5a) ${ }^{65,73}$ When NP-amide was mixed with PBA in methanol- $\mathrm{d}_{4}$, we observed the peak for the boronic ester at $25.1 \mathrm{ppm}$, along with an additional peak at $7.6 \mathrm{ppm}$ (Figure 5c). When PBA was replaced with a more electron-deficient boronic acid, para-nitrophenylboronic acid (NPBA), this peak slightly increased in intensity (Figure 5d), indicating that removing electron density from boron favors the formation of this species. This minor peak did not appear when PBA and NP-amide are combined in $\mathrm{CDCl}_{3}$, a solvent that cannot undergo insertion (Figure 5e). Furthermore, the combination of NP and PBA in methanol- $\mathrm{d}_{4}$ did not reveal any solvent-inserted species (Figure 5f). Taken together, these data suggest that the amide bond can interact with the boronic ester via solvent insertion (Figure 5a, bold box). While the ${ }^{11} \mathrm{~B}$ NMR experiment in methanol- $\mathrm{d}_{4}$ does not replicate the conditions used for kinetics and rheology, and the stability of the solvent-inserted species may differ under those conditions, these experiments suggest that the carbonyl is sufficiently basic and suitably positioned to act as an internal base catalyst during alcohol and water attack. 
(a)

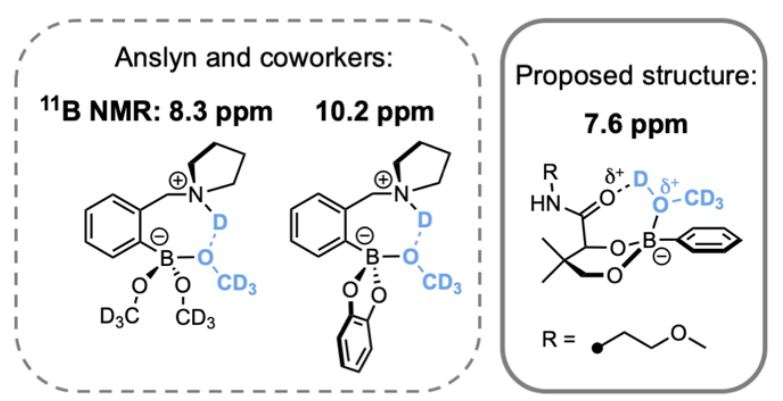

(b) $\mathrm{PBA}$ in $\mathrm{CD}_{3} \mathrm{OD}$

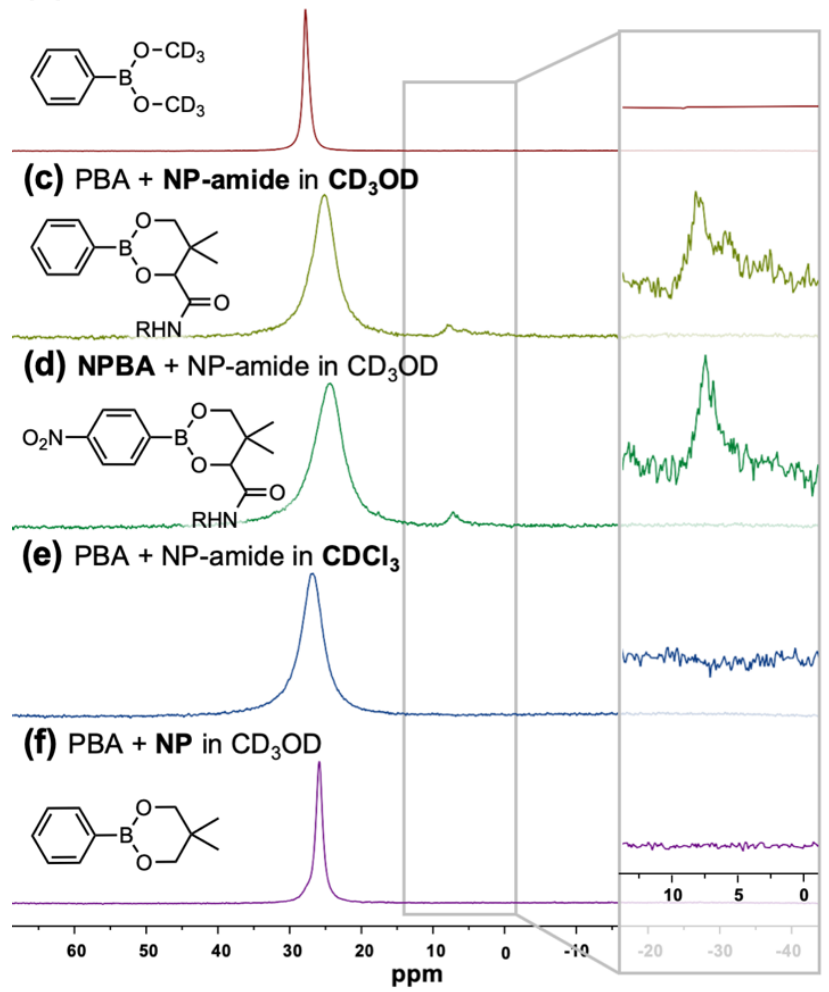

Figure 5. ${ }^{11}$ B NMR study with NP-amide and PBA. Inset shows the zoomed region from 0 to $14 \mathrm{ppm}$. (a) Reported solvent-inserted species of a Wulff-type boronic acid in methanol- $\mathrm{d}_{4}$ with ${ }^{11} \mathrm{~B}$ NMR assignments by Anslyn (left, in the dotted box $)^{65}$ and proposed structure of the solvent-inserted species formed in the presence of an amide linker (right, in the bold box). (b) PBA in $\mathrm{CD}_{3} \mathrm{OD}$ (c) PBA with 10 equiv. NP-amide in $\mathrm{CD}_{3}$ OD. (d) NPBA with 10 equiv. NP-amide in $\mathrm{CD}_{3} \mathrm{OD}$. (e) PBA with 10 equiv. NP-amide in $\mathrm{CDCl}_{3}$. (f) PBA with 10 equiv. NP in $\mathrm{CD}_{3} \mathrm{OD}$.

\section{Amide derivatives better predict the trend in network properties}

The amide derivatives exhibited more pronounced rate acceleration for hydrolysis than for esterification, except for NP-amide. As a result, the binding constants for Tris-amide, CP-amide, and Glu-amide with PBA are lower than those of their simplified alcohol counterparts. Therefore, when the amide effect is considered, the trend in binding affinities for the amide model systems (Tris-amide $<$ NP-amide $<$ CP- 
amide $<$ Glu-amide) is consistent with the trend in plateau modulus observed for the gels (PEG-Tris $<$ PEG-NP $<$ PEG-CP $<$ PEG-Glu). Furthermore, based on the rate constants for the amide derivatives, the self-healing rates of the networks are qualitatively consistent with the $k_{\mathrm{obs}, \mathrm{f}}$ trends. Of the derivatives tested, PEG-Tris exhibits the fastest self-healing, consistent with Tris-amide having the highest $k_{\mathrm{obs}, \mathrm{f}}$.

The $k_{\text {obs,r }}$ values predict a trend in bulk relaxation times (Tris-amide $<$ NP-amide $<$ Glu-amide $<$ (anti)CP-amide) that more closely resembles the gel data (PEG-Tris $<$ PEG-Glu $<$ PEG-NP $<$ PEG-CP). The ratio of anti:syn isomers in PEG-CP affects the relaxation time, with a greater fraction of syn isomer (anti:syn $=1.5: 1$ vs. $4: 1)$ corresponding to faster stress relaxation $(\tau=355$ vs. $571 \mathrm{~s})$, again providing evidence for the relevance of internal catalysis in the mechanism by which the network relaxes stress (Figure S36). We note that while the measured rate constant for Glu-amide hydrolysis is $\sim 3$-fold slower than NP-amide hydrolysis based on our EXSY experiments, PEG-Glu relaxes stress $\sim 2$-fold faster than PEG-NP. However, this discrepancy may be ascribed in part to the buildup of an intermediate in the dynamic reaction of Glu-amide, which may affect the rate constants measured by EXSY.

Overall, our data agree with the paradigm first articulated by Craig for dynamic networks: "strong means slow", meaning that the gels that resist flow have slower kinetics for the crosslink-breaking step. ${ }^{26}$ Previous studies of supramolecular networks by Craig and Scherman have shown that the frequency sweeps for chemically distinct networks can be superimposed when scaled to a molecular parameter such as $k_{\mathrm{r}}$ or $E_{\mathrm{a}, \mathrm{r}}$ ("time-crosslinker superposition"). ${ }^{24,74}$ However, those metal-ligand and host-guest systems undergo flow through single-step, dissociative mechanisms. When comparing our boronic ester networks, $1 / k_{\text {obs-r }}$ and $\tau$ do not scale exactly, and it was not possible to superimpose the frequency sweep data by applying $1 / k_{\mathrm{obs}, \mathrm{r}}$ as horizontal shift factors (Figure S35). We ascribe these differences to the complexity of boronic ester hydrolysis, a multi-step mechanism in which the dominant pathway and speciation can change depending on the structure of the boronic acid and diol, and environmental factors like solvent and $\mathrm{pH} .{ }^{\S, 50,54,62}$ Consistent with changes in mechanism or rate-limiting step, the small-molecule activation energies do not display any trends, and are not consistently lowered by the presence of an internal catalyst (e.g. $E_{\mathrm{a}, \mathrm{r}}$ is 20 $\mathrm{kJ} / \mathrm{mol}$ for Tris and $36 \mathrm{~kJ} / \mathrm{mol}$ for Tris-amide; see Table S3).

\section{External catalysis by buffering anions}

Many boronic ester-based hydrogels are designed for biomedical applications, so are typically formed and studied in the presence of buffers or media containing inorganic salts, amino acids, glucose, and vitamins. While previous studies have focused on the $\mathrm{pH}$ of the medium rather than the buffer itself, Springsteen and Wang showed that the binding affinity of a catechol dye to PBA is sensitive to phosphate buffer composition and concentration. ${ }^{75}$ Furthermore, London and Gabel showed that the presence of phosphate anion increased the dissociation rate of 4-fluorophenylboronic esters. ${ }^{76}$ In the course of our studies, we also observed significant changes in viscoelasticity of gels based on the identity and concentration of buffer salts. Therefore, we used 1D EXSY technique to systematically determine the effect of ions on the rate of boronic ester hydrolysis. NP was used as the model diol for this study, and various salts were introduced to the solution at a final concentration of $0.0092 \mathrm{M}$, due to solubility limitations. To eliminate any changes in rate due to $\mathrm{pH}$, the $\mathrm{pH}$ of each solution was adjusted to 7 using either $\mathrm{DCl}$ or $\mathrm{NaOD}$ as necessary.

The pseudo-first-order rate constants for esterification and hydrolysis in the presence of various salts are shown in Table 2. Using a common buffer, phosphate-buffered saline (PBS), we observed two orders of magnitude increase of the rate constants for both the forward and reverse reactions. The effect of phosphate anion was further confirmed by testing a phosphate salt with a different cation $\left(\mathrm{NBu}_{4} \mathrm{H}_{2} \mathrm{PO}_{4}\right)$, which

revealed very similar rate acceleration. London and Gabel proposed that phosphate anions catalyze proton 
transfer from water during hydrolysis. ${ }^{76}$ We further observed that the extent of acceleration was correlated with the $\mathrm{pKa}$ of the conjugate acid: KOAc provides $\sim 7$-fold acceleration, compared to $\mathrm{D}_{2} \mathrm{O}$ alone, and $\mathrm{Na}_{2} \mathrm{SO}_{4}$ and $\mathrm{NBu}_{4} \mathrm{PF}_{6}$ show no effect. We also did not see any effect in the presence of $\mathrm{NaCl}$ or $\mathrm{CaCl}_{2}$, indicating that the rate acceleration does not originate in ionic strength of the medium.

Table 2. Rate constants for the reversible esterification/hydrolysis of PBA with NP in the presence of different salts at $\mathrm{pH} 7 .^{a}$

\begin{tabular}{|c|c|c|c|}
\hline Salt & $\mathbf{p K} \mathbf{a}^{b}$ & $k_{\text {obs }, \mathrm{f}}\left(\mathbf{s}^{-1}\right)$ & $k_{\text {obs, },}\left(\mathbf{s}^{-1}\right)$ \\
\hline No salt & - & $0.0473( \pm 0.0004)$ & $0.082( \pm 0.001)$ \\
\hline $\mathrm{PBS}\left(\mathrm{NaH}_{2} \mathrm{PO}_{4}+\mathrm{Na}_{2} \mathrm{HPO}_{4}\right)$ & \multirow{2}{*}{$\mathrm{H}_{2} \mathrm{PO}_{4}^{-}=7.21$} & $5.59( \pm 0.07)$ & $8.2( \pm 0.1)$ \\
\hline $\mathrm{NBu}_{4} \mathrm{H}_{2} \mathrm{PO}_{4}$ & & $4.90( \pm 0.06)$ & $7.9( \pm 0.1)$ \\
\hline KOAC & $\mathrm{CH}_{3} \mathrm{COOH}=4.76$ & $0.371( \pm 0.002)$ & $0.574( \pm 0.005)$ \\
\hline $\mathrm{Na}_{2} \mathrm{SO}_{4}$ & $\mathrm{HSO}_{4}^{-}=1.9$ & $0.053( \pm 0.002)$ & $0.087( \pm 0.004)$ \\
\hline $\mathrm{NBu}_{4} \mathrm{PF}_{6}$ & - & $0.0587( \pm 0.0006)$ & $0.095( \pm 0.001)$ \\
\hline $\mathrm{NaCl}$ & \multirow{2}{*}{$\mathrm{HCl}=-6.3$} & $0.051( \pm 0.001)$ & $0.081( \pm 0.002)$ \\
\hline $\mathrm{CaCl}_{2}$ & & $0.052( \pm 0.001)$ & $0.084( \pm 0.003)$ \\
\hline
\end{tabular}

${ }^{a}$ Pseudo-first-order rate constants $k_{\mathrm{obs}, \mathrm{f}}$ and $k_{\mathrm{obs}, \mathrm{r}}$ were measured at $25^{\circ} \mathrm{C}$ by $1 \mathrm{D}$ EXSY with PBA $(19 \mathrm{mM})$ in the presence of 10 equiv. NP and salts $(0.0092 \mathrm{M})$ in acetone- $\mathrm{d}_{6}: \mathrm{D}_{2} \mathrm{O}(1.2: 1)$. The $\mathrm{pH}$ of the solutions was adjusted to 7 with either $\mathrm{DCl}$ or $\mathrm{NaOD}$, if necessary. ${ }^{b}$ From pKa data compiled by R. Williams. ${ }^{* *}$

We anticipated that the dramatic effects on hydrolysis rate would also translate to the viscoelasticity of boronic ester gels. Indeed, the characteristic relaxation time for the PEG-NP gel in the absence of any salts is 100 times slower than that of the same gel with $0.01 \mathrm{M} \mathrm{KOAc}$, which in turn is 10 times slower than the gel with $0.01 \mathrm{M} \mathrm{NBu}_{4} \mathrm{H}_{2} \mathrm{PO}_{4}$ (Figure 6a-b). This 1000 -fold range in relaxation times is achieved solely through the addition of salts, without any change in $\mathrm{pH}$. Visually, it is apparent that the network forms a self-supporting gel without salt, while it is a viscoelastic liquid with $0.01 \mathrm{M} \mathrm{NBu}_{4} \mathrm{H}_{2} \mathrm{PO}_{4}$ (Figure 6c). As the gels became faster-relaxing, the rubbery plateau moduli also decreased (Figure 6b), consistent with the effect of the salts on $K_{\text {eq }}$ in propylene carbonate (Figure S41). ${ }^{\S}$ 
(a)

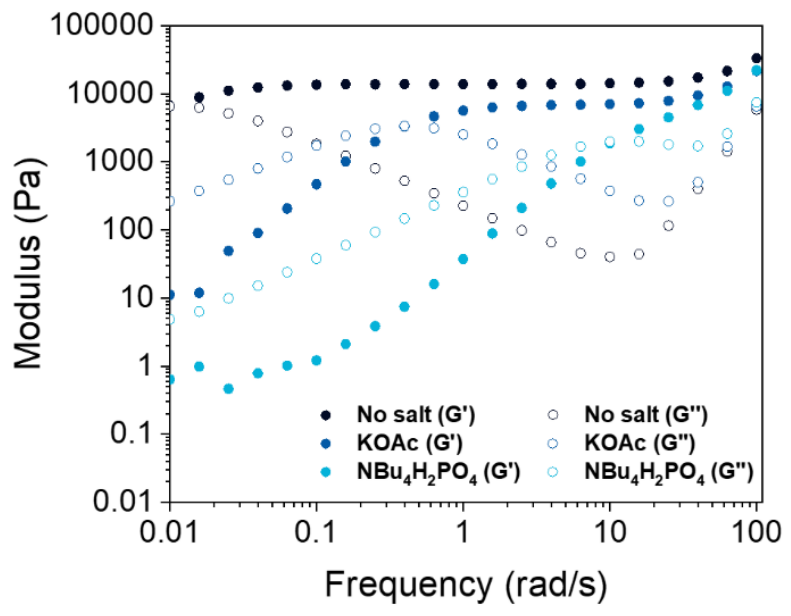

(b)

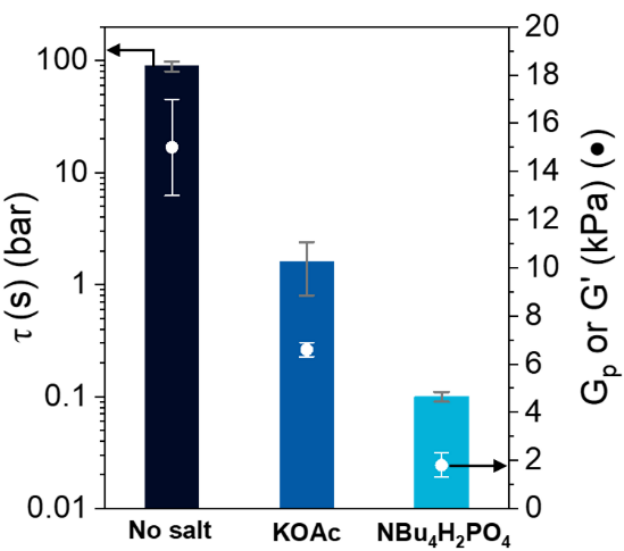

(c)

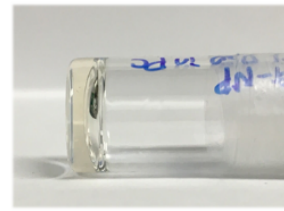

No salts

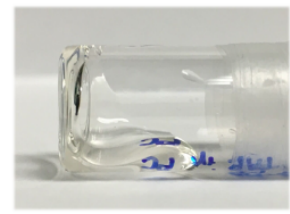

$+0.01 \mathrm{M} \mathrm{NBu}_{4} \mathrm{H}_{2} \mathrm{PO}_{4}$

Figure 6. Effects of anion coordination on the mechanical properties of the PEG-NP gels $(10 \mathrm{w} / \mathrm{v} \%$ in propylene carbonate with $1 \% 1 \mathrm{M}$ salt in $\mathrm{D}_{2} \mathrm{O}$ ) formed with solution containing different salts (final concentration of salt: $0.01 \mathrm{M}$ ). $\mathrm{pH}$ of the $1 \mathrm{M}$ stock solution of the salt in $\mathrm{D}_{2} \mathrm{O}$ was adjusted to 7 with either $\mathrm{DCl}$ or $\mathrm{NaOD}$. (a) Frequency sweep with $5 \%$ strain. (b) Calculated $\tau$ (bar graph, left axis) and the rubbery plateau modulus, $\mathrm{G}_{\mathrm{p}}$ (dot, right axis). In the case of the viscoelastic liquid formed with $\mathrm{NBu}_{4} \mathrm{H}_{2} \mathrm{PO}_{4}, \mathrm{G}^{\prime}$ at $10 \mathrm{rad} / \mathrm{s}$ was used as a plateau modulus is not observed. Mechanical data were obtained on 3 samples; error is the standard deviation of the mean. (c) Photographs of PEG-NP gels at $\mathrm{pH} 7$ in the absence and the presence of $0.01 \mathrm{M} \mathrm{NBu} \mathrm{H}_{2} \mathrm{PO}_{4}$ salts.

\section{Conclusion}


Small-molecule model studies, including our own, ${ }^{13}$ are often performed with simple diols like ethylene glycol or fructose in organic solvents such as DMSO, which simplifies analysis. In comparison, the attachment of diols to polymers requires the installation of non-innocent functional groups like amides. Furthermore, the resulting materials are often studied in complex aqueous environments like buffer or media. Our findings suggest that small-molecule model studies based on simple diols in organic solvents may not sufficiently capture the behavior of materials, particularly in the translation from molecular kinetics to bulk dynamic mechanical properties.

By comparing the small-molecule kinetics of dynamic covalent reactions to the macroscopic properties of covalent adaptable networks, we have discovered two factors that affect gel mechanics by accelerating boronic acid esterification and hydrolysis: proximal amides commonly used to conjugate crosslinkers to polymers, and anions found in buffers and media. The extent of this rate acceleration can be precisely tuned through the position of the amide relative to the diol and the basicity of the anion, and therefore can be viewed as tunable design parameters for dynamic materials. In boronic ester-based gels, the accelerated hydrolysis rate translates into faster stress relaxation. Accelerated esterification analogously translates to faster self-healing. Thus, linkage chemistry and buffer composition are essential parameters to consider for applications of this dynamic bond to stimuli-responsive and self-healing materials. The internal catalysis effect may extend to other Lewis basic groups used for polymer modification, such as triazoles ${ }^{49}$ or oximes. ${ }^{77}$ We have also shown that internal or external catalysis affects the stiffness of the polymer network because rate acceleration is generally more pronounced for hydrolysis than for esterification. These effects could be exploited in the design of dynamic materials that change physical properties in response to anions or diols. We envision this work will contribute to a growing understanding of the boronic ester dynamic bond and provide strategies to molecularly engineer dynamic materials.

\section{Acknowledgements}

The small-molecule studies in this work were supported by the National Science Foundation under CAREER award CHE-1847948, and the network studies were supported by the National Institute of General Medical Sciences of the National Institutes of Health under award number R01GM132677. This content is solely the responsibility of the authors and does not necessarily represent the official views of the National Science Foundation or National Institutes of Health. J.A.K. is supported by a Sloan Research Fellowship and a Dreyfus Teacher-Scholar Award. This work made use of NMR and MS instrumentation at the Integrated Molecular Structure Education and Research Center (IMSERC) at Northwestern, which has received support from the NSF (NSF CHE-9871268); Soft and Hybrid Nanotechnology Experimental (SHyNE) Resource (NSF ECCS-1542205); the State of Illinois and International Institute for Nanotechnology. Rheological measurements were performed at the MatCI Facility, which receives support from the MRSEC Program (NSF DMR-1720139) of the Materials Research Center at Northwestern University.

\section{Notes and references}

1. X. Wu, Z. Li, X. X. Chen, J. S. Fossey, T. D. James and Y. B. Jiang, Chem. Soc. Rev., 2013, 42, 8032-8048.

2. Z. Bian, A. Liu, Y. Li, G. Fang, Q. Yao, G. Zhang and Z. Wu, Analyst, 2020, 145, 719-744.

3. W. L. Brooks and B. S. Sumerlin, Chem. Rev., 2016, 116, 1375-1397.

4. E. Galbraith and T. D. James, Chem. Soc. Rev., 2010, 39, 3831-3842.

5. Y. Guan and Y. Zhang, Chem. Soc. Rev., 2013, 42, 8106-8121.

6. A. Stubelius, S. Lee and A. Almutairi, Acc. Chem. Res., 2019, 52, 3108-3119. 
7. S. Tang, H. Ma, H. Tu, H. Wang, P. Lin and K. S. Anseth, Adv. Sci., 2018, 5, 1800638.

8. J. V. Accardo and J. A. Kalow, Chem. Sci., 2018, 9, 5987-5993.

9. C. C. Deng, W. L. A. Brooks, K. A. Abboud and B. S. Sumerlin, ACS Macro Lett., 2015, 4, 220-224.

10. M. E. Smithmyer, C. C. Deng, S. E. Cassel, P. J. LeValley, B. S. Sumerlin and A. M. Kloxin, ACS Macro Lett., 2018, 7, 1105-1110.

11. Z. Huang, P. Delparastan, P. Burch, J. Cheng, Y. Cao and P. B. Messersmith, Biomater. Sci., 2018, 6, 2487-2495.

12. A. Pettignano, S. Grijalvo, M. Haring, R. Eritja, N. Tanchoux, F. Quignard and D. Diaz Diaz, Chem. Commun., 2017, 53, 3350-3353.

13. J. V. Accardo, E. R. McClure, M. A. Mosquera and J. A. Kalow, J. Am. Chem. Soc., 2020, 142, 19969-19979.

14. S. H. Hong, S. Kim, J. P. Park, M. Shin, K. Kim, J. H. Ryu and H. Lee, Biomacromolecules, 2018, 19, 2053-2061.

15. B. Marco-Dufort, J. Willi, F. Vielba-Gomez, F. Gatti and M. W. Tibbitt, Biomacromolecules, 2021, 22, 146-157.

16. J. N. Cambre, D. Roy and B. S. Sumerlin, J. Polym. Sci. A: Polym. Chem., 2012, 50, 3373-3382.

17. D. Roy and B. S. Sumerlin, ACS Macro Lett., 2012, 1, 529-532.

18. T. Figueiredo, J. Jing, I. Jeacomine, J. Olsson, T. Gerfaud, J. G. Boiteau, C. Rome, C. Harris and R. Auzely-Velty, Biomacromolecules, 2020, 21, 230-239.

19. M. M. de Oliveira, C. V. Nakamura and R. Auzely-Velty, Carbohydr. Polym., 2020, 247, 116845.

20. T. Figueiredo, Y. Ogawa, J. Jing, V. Cosenza, I. Jeacomine, J. D. M. Olsson, T. Gerfaud, J.-G. Boiteau, C. Harris and R. Auzély-Velty, Polym. Chem., 2020, 11, 3800-3811.

21. D. Roy, J. N. Cambre and B. S. Sumerlin, Chem. Commun., 2009, DOI: 10.1039/b900374f, 2106-2108.

22. S. Tang, B. M. Richardson and K. S. Anseth, Prog. Mater. Sci., 2021, 120.

23. L. L. Robinson, J. L. Self, A. D. Fusi, M. W. Bates, J. Read de Alaniz, C. J. Hawker, C. M. Bates and C. S. Sample, ACS Macro Lett., 2021, 10, 857-863.

24. W. C. Yount, D. M. Loveless and S. L. Craig, J. Am. Chem. Soc., 2005, 127, 14488-14496.

25. R. J. Sheridan and C. N. Bowman, Macromolecules, 2012, 45, 7634-7641.

26. W. C. Yount, D. M. Loveless and S. L. Craig, Angew. Chem. Int. Ed., 2005, 44, 2746-2748.

27. O. R. Cromwell, J. Chung and Z. Guan, J. Am. Chem. Soc., 2015, 137, 6492-6495.

28. T. Figueiredo, V. Cosenza, Y. Ogawa, I. Jeacomine, A. Vallet, S. Ortega, R. Michel, J. D. M. Olsson, T. Gerfaud, J. G. Boiteau, J. Jing, C. Harris and R. Auzely-Velty, Soft Matter, 2020, 16, 3628-3641.

29. S. Tang and B. D. Olsen, Macromolecules, 2016, 49, 9163-9175.

30. C. S. Y. Tan, G. Agmon, J. Liu, D. Hoogland, E.-R. Janeček, E. A. Appel and O. A. Scherman, Polym. Chem., 2017, 8, 5336-5343.

31. A. N. Semenov and M. Rubinstein, Macromolecules, 1998, 31, 1373-1385.

32. M. Rubinstein and A. N. Semenov, Macromolecules, 1998, 31, 1386-1397.

33. B. Marco-Dufort, R. Iten and M. W. Tibbitt, J. Am. Chem. Soc., 2020, 142, 15371-15385.

34. X. Kuang, G. Liu, X. Dong and D. Wang, Mater. Chem. Front., 2017, 1, 111-118.

35. G. A. Parada and X. Zhao, Soft Matter, 2018, 14, 5186-5196.

36. D. D. McKinnon, D. W. Domaille, J. N. Cha and K. S. Anseth, Chem. Mater., 2014, 26, 2382-2387.

37. T. M. FitzSimons, F. Oentoro, T. V. Shanbhag, E. V. Anslyn and A. M. Rosales, Macromolecules, 2020, 53, 3738-3746.

38. M. Guerre, C. Taplan, J. M. Winne and F. E. Du Prez, Chem. Sci., 2020, 11, 4855-4870.

39. N. Van Herck, D. Maes, K. Unal, M. Guerre, J. M. Winne and F. E. Du Prez, Angew. Chem. Int. Ed., 2020, 59, 3609-3617.

40. M. Delahaye, J. M. Winne and F. E. Du Prez, J. Am. Chem. Soc., 2019, 141, 15277-15287.

41. B. Marco-Dufort and M. W. Tibbitt, Mater. Today Chem., 2019, 12, 16-33.

42. J. Yan, G. Springsteen, S. Deeter and B. Wang, Tetrahedron, 2004, 60, 11205-11209.

43. C. D. Roy and H. C. Brown, J. Organomet. Chem., 2007, 692, 784-790.

44. M. Dowlut and D. G. Hall, J. Am. Chem. Soc., 2006, 128, 4226-4227.

45. L. I. Bosch, T. M. Fyles and T. D. James, Tetrahedron, 2004, 60, 11175-11190.

46. J. A. Peters, Coord. Chem. Rev., 2014, 268, 1-22.

47. W. L. A. Brooks, C. C. Deng and B. S. Sumerlin, ACS Omega, 2018, 3, 17863-17870.

48. Y. Cheng, N. Ni, W. Yang and B. Wang, Chem. Eur. J., 2010, 16, 13528-13538.

49. M. Lin, P. Sun, G. Chen and M. Jiang, Chem. Commun., 2014, 50, 9779-9782.

50. G. Wulff, M. Lauer and H. Böhnke, Angew. Chem. Int. Ed., 1984, 23, 741-742.

51. T. Matsumura, S. Iwatsuki and K. Ishihara, Inorg. Chem. Commun., 2005, 8, 713-716.

52. B. E. Collins, P. Metola and E. V. Anslyn, Supramol. Chem., 2013, 25, 79-86.

53. J. W. Tomsho and S. J. Benkovic, J. Org. Chem., 2012, 77, 2098-2106. 
54. Y. Furikado, T. Nagahata, T. Okamoto, T. Sugaya, S. Iwatsuki, M. Inamo, H. D. Takagi, A. Odani and K. Ishihara, Chem. Eur. J., 2014, 20, 13194-13202.

55. E. Watanabe, C. Miyamoto, A. Tanaka, K. Iizuka, S. Iwatsuki, M. Inamo, H. D. Takagi and K. Ishihara, Dalton Trans., 2013, 42, 8446-8453.

56. R. Pizer and C. Tihal, Inorg. Chem., 1992, 3243-3247.

57. H. Monajemi, M. H. Cheah, V. S. Lee, S. M. Zain and W. A. Tajuddin Wan Abdullah, RSC Adv., $2014,4$.

58. C. Miyamoto, K. Suzuki, S. Iwatsuki, M. Inamo, H. D. Takagi and K. Ishihara, Inorg. Chem., 2008, 47, 14171419.

59. H. Ito, Y. Kono, A. Machida, Y. Mitsumoto, K. Omori, N. Nakamura, Y. Kondo and K. Ishihara, Inorganica Chim. Acta, 2003, 344, 28-36.

60. R. D. Pizer and C. A. Tihal, Polyhedron, 1996, 15, 3411-3416.

61. N. Ni, S. Laughlin, Y. Wang, Y. Feng, Y. Zheng and B. Wang, Bioorg. Med. Chem., 2012, 20, $2957-2961$.

62. Y. Suzuki, D. Kusuyama, T. Sugaya, S. Iwatsuki, M. Inamo, H. D. Takagi and K. Ishihara, J. Org. Chem., 2020, 85, 5255-5264.

63. L. Babcock and R. Pizer, Inorg. Chem., 1980, 19, 56-61.

64. R. Pizer and L. Babcock, Inorg. Chem., 1977, 16, 1677-1681.

65. L. Zhu, S. H. Shabbir, M. Gray, V. M. Lynch, S. Sorey and E. V. Anslyn, J. Am. Chem. Soc., 2006, 128, 12221232.

66. A. Swartjes, P. B. White, M. Lammertink, J. Elemans and R. J. M. Nolte, Angew. Chem. Int. Ed., 2021, 60, 12541262.

67. D. Barsoum and J. Kalow, 2021, DOI: 10.33774/chemrxiv-2021-lwk72.

68. X. Sun, B. M. Chapin, P. Metola, B. Collins, B. Wang, T. D. James and E. V. Anslyn, Nat. Chem., 2019, 11, 768778.

69. J. Yoshino, N. Kano and T. Kawashima, Tetrahedron, 2008, 64, 7774-7781.

70. N. Kano, J. Yoshino and T. Kawashima, Org. Lett., 2005, 7, 3909-3911.

71. H. Chen, H. Ye, Y. Hai, L. Zhang and L. You, Chem. Sci., 2020, 11, 2707-2715.

72. C. Hansch, A. Leo and R. W. Taft, Chem. Rev., 2002, 91, 165-195.

73. B. E. Collins, S. Sorey, A. E. Hargrove, S. H. Shabbir, V. M. Lynch and E. V. Anslyn, J. Org. Chem., 2009, 74, 4055-4060.

74. E. A. Appel, R. A. Forster, A. Koutsioubas, C. Toprakcioglu and O. A. Scherman, Angew. Chem. Int. Ed., 2014, 53, 10038-10043.

75. G. Springsteen and B. Wang, Tetrahedron, 2002, 58, 5291-5300.

76. R. E. London and S. A. Gabel, J. Am. Chem. Soc., 1994, 116, 2562-2569.

77. D. Tarus, E. Hachet, L. Messager, B. Catargi, V. Ravaine and R. Auzely-Velty, Macromol. Rapid. Commun., 2014, 35, 2089-2095.

\footnotetext{
$\S$ Despite similar structures for the solvents used for EXSY (acetone) and rheology (propylene carbonate), some differences are observed for binding constants in each solvent, which could contribute to the absence of timecrosslinker superposition. For more details, see Figure S41.

** Online access: https://organicchemistrydata.org/hansreich/resources/pka/pka_data/pka-compilation-williams.pdf
} 